\title{
Ambiguous definitions for baseline serum creatinine affect acute kidney diagnosis at the emergency department
}

Michael Niemantsverdriet ${ }^{1,2}$ Meriem Khairoun ${ }^{3}$, Ayman El Idrissi' ${ }^{4}$, Romy Koopsen ${ }^{4}$, Imo Hoefer', Wouter van Solinge ${ }^{1}$, Jan Willem Uffen ${ }^{4}$, Domenico Bellomo ${ }^{2}$, Wouter Tiel Groenestege ${ }^{1}$, Karin Kaasjager ${ }^{4}$ and Saskia Haitjema ${ }^{1 *}$

\begin{abstract}
Background: Acute kidney injury (AKI) incidence is increasing, however AKI is often missed at the emergency department (ED). AKI diagnosis depends on changes in kidney function by comparing a serum creatinine ( $\mathrm{SCr}$ ) measurement to a baseline value. However, it remains unclear to what extent different baseline values may affect AKI diagnosis at ED.

Methods: Routine care data from ED visits between 2012 and 2019 were extracted from the Utrecht Patient Oriented Database. We evaluated baseline definitions with criteria from the RIFLE, AKIN and KDIGO guidelines. We evaluated four baseline SCr definitions (lowest, most recent, mean, median), as well as five different time windows (up to 365 days prior to ED visit) to select a baseline and compared this to the first measured SCr at ED. As an outcome, we assessed AKI prevalence at ED.

Results: We included 47,373 ED visits with both SCr-ED and SCr-BL available. Of these, 46,100 visits had a SCr-BL from the $-365 /-7$ days time window. Apart from the lowest value, AKI prevalence remained similar for the other definitions when varying the time window. The lowest value with the $-365 /-7$ time window resulted in the highest prevalence (21.4\%). Importantly, applying the guidelines with all criteria resulted in major differences in prevalence ranging from 5.9 to $24.0 \%$.

Conclusions: AKI prevalence varies with the use of different baseline definitions in ED patients. Clinicians, as well as researchers and developers of automatic diagnostic tools should take these considerations into account when aiming to diagnose AKI in clinical and research settings.
\end{abstract}

Keywords: AKI, Creatinine, CKD-EPI, Electronic health records, Digital health

\section{Background}

Acute kidney injury (AKI) is the most common complication in hospitalized patients and is associated with high morbidity and mortality [1]. The incidence of AKI

\footnotetext{
*Correspondence: S.Haitjema@umcutrecht.nl

${ }^{1}$ Central Diagnostic Laboratory, University Medical Center Utrecht, Utrecht University, Room Number G03.551, UMC Utrecht, Heidelberglaan 100, Utrecht 3584 CX, The Netherlands

Full list of author information is available at the end of the article
}

is increasing due to the growing incidence of risk factors, including cardiovascular disease, use of nephrotoxic medication and contrast-containing imaging [2]. As mild increases of serum creatinine $(\mathrm{SCr})$ are associated with adverse outcome, it's important to identify risk factors and to increase awareness of AKI in healthcare systems [3]. However, AKI is often missed, due to lack of awareness and knowledge on early recognition, prevention and management of AKI by physicians from different 
specialties [4]. Importantly, recent studies indicated that early detection of AKI improves short and long-term outcomes [5].

To improve awareness and promote early detection by health care providers, multiple guidelines have been developed, including Risk, Injury, Failure, Loss and Endstage (RIFLE), Acute Kidney Injury Network (AKIN) and Kidney Disease Improving Global Outcomes (KDIGO), as this may lead to initiation of early interventions, such as adequate fluid management, adjustment of medication dose and avoiding the use of specific nephrotoxins [6-8]. Overall, these guidelines postulate criteria for AKI diagnosis by detecting changes in endogenous surrogate markers of kidney function, such as $\mathrm{SCr}$ and urine output [9].

Growing use of electronic health records (EHR) and machine learning have provided a possibility to study large collections of real-world data and develop early detection systems for AKI [10]. Indeed, clinical decision support systems (CDS) have emerged as tools for initial assessment and identification of AKI patients in different settings [11]. These CDS make recommendations and risk stratifications based on the existing guidelines and best practices for AKI [12]. Understanding the specific definitions of the guidelines and their implications on AKI diagnosis is thus of utmost importance in both patient care and research.

In brief, the diagnostic criteria for AKI are based on a change between a current $\mathrm{SCr}$ and a previous $\mathrm{SCr}$ measurement known as 'baseline' in the preceding days or period. As kidney function of hospitalized patients is routinely monitored, $\mathrm{SCr}$ measurements during admission are often available to compare with the baseline value, usually defined as the first measurement during admission or at ED presentation. However, patients who visit the ED may lack $\mathrm{SCr}$ measurements from the preadmission period, making the criteria less suitable for this setting. As a consequence, several studies have proposed multiple ways to define baseline using different values and time windows [13].

Multiple definitions have been used in literature to select a baseline value from the patient's clinical history, such as the most recent value, the median value, the mean or the lowest value [14-16]. Depending on available data, researchers have applied varying lengths for the baseline time window and baseline definitions for AKI diagnosis [17]. The use of different definitions has been shown to affect AKI prevalence in hospitalized patients [13]. However, to which extent the use of various baseline definition and time windows influence AKI diagnosis in the ED has not been investigated.

Here, we systematically compared various baseline definitions using AKI criteria from the RIFLE, AKIN and
KDIGO guidelines with several combinations of baseline time window and baseline definition to assess the effect on AKI diagnosis, using prevalence as an outcome, in a large cohort of ED patients from our center.

\section{Methods \\ Study population}

We performed a single center retrospective analysis, using routine care data from the University Medical Center Utrecht (UMCU), a large tertiary referral center in Utrecht, the Netherlands. All ED visits between 2012 and 2019 from adult patients over 18 years of age were included. Data was extracted from the Utrecht Patient Orientated Database (UPOD). In brief, UPOD is an infrastructure of relational databases comprising data on patient characteristics, hospital discharge diagnoses, medical procedures, medication orders and laboratory tests for all patients treated at the UMCU since 2004 [18].

From UPOD, for each ED visit we included patient's age, gender and treating specialty. Additionally, all $\mathrm{SCr}$ measurements were extracted 365 days prior to ED visit, as well as the first measurement at ED. We defined the latter as the first measurement within $6 \mathrm{~h}$ after ED admission, as the majority of ED visits are either send home or admitted to the hospital within hours after ED admission (SCr-ED). ED visits with no SCr measurements 365 days prior to ED visit and a SCr-ED were excluded from this study. $\mathrm{SCr}$ was measured by isotope dilution mass spectrometry traceable enzymatic colorimetric assays (Beckman Coulter, Brea, CA, USA and Siemens Healthcare Diagnostic Inc., NY, USA). Estimated Glomerular Filtration Rate (eGFR) was calculated by the CKD-EPI formula [19]. Chronic Kidney Disease (CKD) was defined according to the KDIGO criteria based on eGFR.

\section{AKI criteria}

To compare the effect of varying baseline definitions on AKI prevalence in the ED, we applied criteria from the RIFLE, AKIN and KDIGO guidelines. As RIFLE does not specify a baseline time window and KDIGO only specifies a time window of 7 days, we used a window of 365 days to define SCr-BL. We did not include the urine output criteria as this is not available in most hospitals. We defined four $\mathrm{SCr}$ criteria and three eGFR criteria applicable to the ED to determine AKI (Table 1) $[15,20]$. Four of the seven criteria specify a baseline time window: two rely upon a window of 7 days ( $-7 / 0$ days) and two define a window of 1 year to a week prior ED visit $(-365$ / -7 days). The remaining three criteria do not define a specific baseline value, but compute changes in the surrogate marker measurements from the $48 \mathrm{~h}$ prior to the ED visit. Each available measurement from this time window was compared as a baseline to the SCr-ED. 
Table 1 Seven criteria for AKI diagnosis adapted from the RIFLE, AKIN and KDIGO guidelines

\begin{tabular}{|c|c|c|c|c|}
\hline Criteria number & Criteria & RIFLE & AKIN & KDIGO \\
\hline 1 & Rise of $\geq 26.5 \mathrm{SCr} 48 \mathrm{~h}$ prior to ED admission & & V & V \\
\hline 2 & Relative increase of $\geq 1.5 \mathrm{SCr}$ to baseline within $48 \mathrm{~h}$ prior to $\mathrm{ED}$ admission & V & V & V \\
\hline 3 & Relative increase of $\geq 1.5 \mathrm{SCr}$ to baseline within 7 days & V & & V \\
\hline 4 & Relative increase of $\geq 1.5 \mathrm{SCr}$ to baseline within 1 year & V & & V \\
\hline 5 & Relative decrease of $>25 \%$ eGFR $48 \mathrm{~h}$ prior to ED admission & V & & \\
\hline 6 & Relative decrease of $>25 \%$ eGFR to baseline within 7 days & V & & \\
\hline 7 & Relative decrease of $>25 \%$ eGFR to baseline within 1 year & V & & \\
\hline
\end{tabular}

Each criterion compares a specific value extracted from a baseline time window before emergency department (ED) with the value measured at the ED. "V" indicates that the criterion is part of the specific guideline. AKI is diagnosed when at least one of the criteria per guideline are met

\section{Analysis}

As patients can have multiple $\mathrm{SCr}$ measurements within a baseline time window, we evaluated baseline definitions in terms of time window and value. This was done only for the four criteria who specify a baseline in the time window prior to ED visit. For each patient visit, we included all $\mathrm{SCr}$ measurements from the patient's medical history from the baseline time window. Then, from this set of SCr measurements we defined baseline serum creatinine ( $\mathrm{SCr}-\mathrm{BL}$ ) for each criterion by applying the four baseline values: median, mean, most recent and lowest.

Additionally, to further characterize the effect of the time window and baseline value combinations, we looked at the effect on AKI prevalence of a varying time window lengths. More specifically, we looked at the combinations of all the four baseline value options and five time windows ranging from -365 days prior up to 7 days prior to ED presentation $(-365 /-7,-270 /-7,-180 /-7,-90 /-7$, $-45 /-7$ days). For each of these 20 combinations we defined a SCr-BL.

After determining the $\mathrm{SCr}-\mathrm{BL}$ for the four baseline criteria, we calculated the AKI prevalence for each individual criterion with the SCr-ED for every ED visit. In order to use the three eGFR criteria, we calculated the eGFR for each selected $\mathrm{SCr}$ value with the CKD-EPI formula. Subsequently, we compared the AKI prevalence between the three guidelines by computing the remaining three criteria who did not define a baseline time window. AKIN prevalence was only computed for patients with measurements in the $48 \mathrm{~h}$ prior to ED visit. Finally, we computed the AKI prevalence for each guideline. The first stage of AKI was deemed as having AKI for all analyses. Data are presented as means with standard deviations. All data pre-processing and analyses were performed using the $R$ environment (version 3.6.1).

\section{Results \\ Baseline characteristics}

We included 20,488 patients who visited our ED between 2012 and 2019, with both a SCr-ED and a SCr-BL available in their EHR, which corresponded to a total of 47,373 visits (Table 2). $54.9 \%$ of the patients had one ED visit (Supplementary Table 1). Most visits were made by men (53.5\%), with an average age of $59.0 \pm 16.8$ and a mean SCr-ED of $108.3 \pm 133.2$. One third of the visits were patients that were subsequently admitted to the internal medicine department (32.3\%). Of the 47,373 visits, $46,100(97.3 \%)$ had at least one SCr measurement available within the $-365 /-7$ baseline time window, 10,554 $(22.3 \%)$ in the $-7 / 0$ and $3322(7.0 \%)$ in the $-2 / 0$ window (not mutually exclusive) (Fig. 1).

\section{Lowest baseline value leads to the highest AKI prevalence} We used four baseline definitions to calculate the delta for both surrogate markers for the $-365 /-7$ and $-7 / 0$ days time windows. We found that the lowest baseline definition resulted in the highest mean delta for both surrogate markers regardless of time window (Supplemental Table 2, Supplemental Figs. 1 and 2). Furthermore, we found a similar effect for the lowest value regardless of time window when we applied the seven criteria on our data (Fig. 2). The eGFR criteria resulted in a higher $\mathrm{AKI}$ prevalence compared to the $\mathrm{SCr}$ criteria (Supplementary Data Table 2). In particular, using the lowest value for both the SCr and the eGFR criteria for the $-365 /-7$ baseline time window resulted in the highest relative AKI prevalence, 15.8 and $21.4 \%$ respectively (Supplemental Table 3).

\section{Reducing the baseline time window width decreases AKI prevalence}

Next, we evaluated the effect of reducing the baseline time window from $-365-7$ to $-45 /-7$ for both surrogate makers (criteria 4 and 7 ). Adjusting the time 
Table 2 Baseline characteristics of all filtered emergency department visits

\begin{tabular}{|c|c|}
\hline & $N=47,373$ ED visits \\
\hline Age, years mean (SD) & $59.0(16.8)$ \\
\hline Male sex, count (\%) & $23,358(53.5 \%)$ \\
\hline Hospitalized, count (\%) & $29,633(62.6 \%)$ \\
\hline \multicolumn{2}{|c|}{ CKD category at ED presentation, $\%(N)$} \\
\hline G1 & $18,246(38.5 \%)$ \\
\hline G2 & $15,491(32.7 \%)$ \\
\hline G3a & $5299(11.2 \%)$ \\
\hline $\mathrm{G} 3 \mathrm{~b}$ & $3965(8.4 \%)$ \\
\hline G4 & $2746(5.8 \%)$ \\
\hline G5 & $1626(3.4 \%)$ \\
\hline \multicolumn{2}{|l|}{ ED specialty, count (\%) } \\
\hline Cardiology & $9003(19.0 \%)$ \\
\hline Gastroenterology & $2885(6.1 \%)$ \\
\hline Internal medicine & $15,233(32.2 \%)$ \\
\hline Pulmonary disease & $5358(11.3 \%)$ \\
\hline Nephrology & $2267(4.8 \%)$ \\
\hline Neurology & $5211(11.0 \%)$ \\
\hline Surgery & $4710(9.9 \%)$ \\
\hline Urology & $2165(4.6 \%)$ \\
\hline Other & $541(1.1 \%)$ \\
\hline $\mathrm{SCr}-\mathrm{ED}, \mu \mathrm{mol} / \mathrm{L}$, mean $(\mathrm{SD})$ & $108.3(113.2)$ \\
\hline \multicolumn{2}{|c|}{$\begin{array}{l}\text { Baseline } \mathrm{SCr} \text { measurements frequency in the previous } 365 \text { days before } \\
\text { ED visit, count (\%) }\end{array}$} \\
\hline $1 \mathrm{SCr}$ measurement & $8710(18.4 \%)$ \\
\hline $2 \mathrm{SCr}$ measurements & $5643(11.9 \%)$ \\
\hline $3 \mathrm{SCr}$ measurements & 4249 (9.0\%) \\
\hline $4 \mathrm{SCr}$ measurements & $3521(7.4 \%)$ \\
\hline $5 \mathrm{SCr}$ measurements & $2792(5.9 \%)$ \\
\hline$>5$ measurements & $22,458(46.9 \%)$ \\
\hline
\end{tabular}

Only emergency department visits with a serum creatinine measurement at emergency department visit and at least one baseline serum creatinine value were selected. Percentages reflect the percentage of the total number of visits. Emergency department specialty was defined as the first specialty the patient encountered during visit

window from $-365 /-7$ to $-45 /-7$ reduced the number of visits with available baseline values from 46,100 to 25,831 (44.0\% decrease). Apart from the lowest baseline value, AKI prevalence remained the same for the mean, median and most recent baseline values when reducing the time window for both $\mathrm{SCr}$ and eGFR criteria. The $\mathrm{SCr}$ criterion with the lowest baseline value in combination with the $-45 /-7$ days time window led to a reduction in AKI prevalence from 15.8 to 9.0\%, whereas the eGFR criterion with the lowest baseline value showed a reduction from 21.4 to $14.5 \%$ (Fig. 3).

\section{RIFLE guidelines yield the highest AKI prevalence}

We compared the RIFLE, AKIN and KDIGO guidelines by applying the guideline-related criteria in combination with the different baseline definitions. To include all ED visits, we used the $-365 /-7$ baseline time window for the two criteria without definition for baseline time window (criteria 4 and 7). Regardless of the used baseline definition (lowest, recent, median or mean), the AKI prevalence was different between the guidelines. Between the three guidelines, we found a maximum difference in AKI prevalence of $23.1 \%$ (range between 5.9 and $24.0 \%$ ) (Table 3). Again, the lowest value resulted in the highest AKI prevalence (RIFLE; 24.0\%, AKIN; 7.7\%, KDIGO; $16.2 \%)$.

\section{Discussion}

In this retrospective study, we evaluated the effect of different baseline definitions on AKI diagnosis at the ED, using prevalence as an outcome. Interestingly, the AKI prevalence varied considerably under different guidelines ranging between 5.7 and $23.6 \%$. Our study shows that selecting the SCr-BL with the lowest baseline definition resulted in the highest AKI prevalence. Furthermore, we also found that expanding the baseline time window length resulted in a higher AKI prevalence for the lowest baseline definition. Moreover, applying the RIFLE guidelines criteria for AKI resulted in the highest prevalence. Our study shows that definition of baseline has important implications for the diagnosis of AKI at the ED.

To our knowledge, this is the first study that performed an in-depth analysis on a large cohort providing insights in the effect of applying various definitions, criteria and guidelines to diagnose AKI at ED. Studies on AKI epidemiology in hospitalized patients have been well described with reported prevalence rates that vary between $<1-66 \%$ [21]. However, studies in the ED population are scarce. Our results are in line with the sparse literature. A previous study in the ED applied the AKIN criteria, corresponding to our $-2 / 0$ baseline time window analysis, and found a prevalence of $6.4 \%$, which is similar to the AKI prevalence of $7.8 \%$ in our study [22]. Another study found an AKI ED prevalence of $5.5 \%$ by applying the KDIGO criteria with a baseline defined 1 year prior ED visit [23]. However, of the studies that compared RIFLE, AKIN and KDIGO for the definition of AKI, none of them investigated the different definitions of baseline with different baseline time-windows [24, 25].

Apart from diagnosing AKI, baseline $\mathrm{SCr}$ is also necessary to evaluate the deterioration of renal function in AKI and to follow the extent of recovery after an AKI event. The methods to estimate baseline $\mathrm{SCr}$ in literature include admission $\mathrm{SCr}$, lowest value prior to admission, minimum $\mathrm{SCr}$ value during hospital admission or a calculation using the MDRD equation in patients without baseline [26]. The definition for baseline $\mathrm{SCr}$ is 


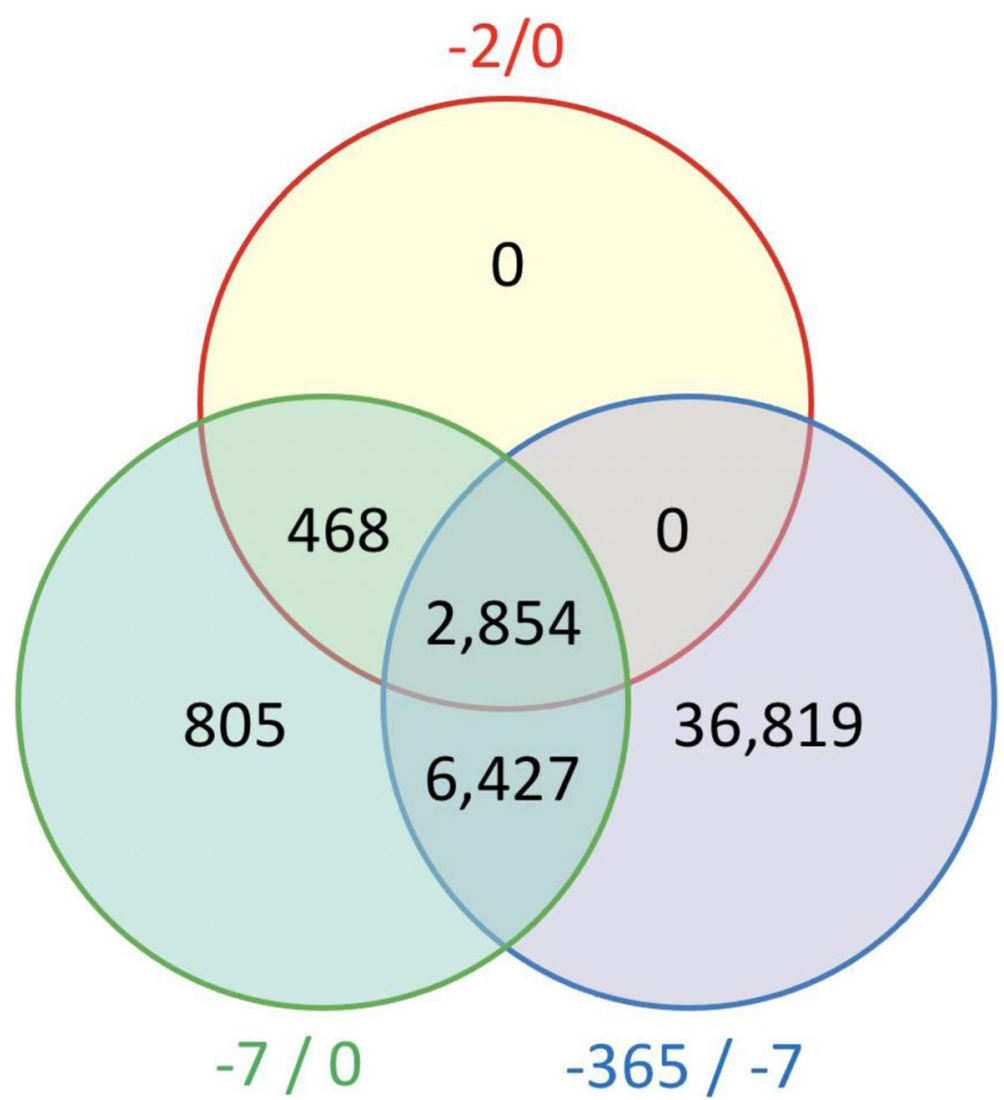

Fig. 1 Number of visits with overlapping serum creatinine measurements from the three baseline time windows

of great importance, since this can significantly affect the assessment of the AKI prevalence and of the associated mortality risk and renal outcomes. Taking the $\mathrm{SCr}$ at admission has been shown to be unrepresentative as baseline, since this value could be influenced by the ongoing disease [27]. On the other hand, using the lowest baseline definition may overestimate AKI prevalence, as shown in this study. As a result, several studies have over- or underestimated AKI diagnosis using different baseline definitions in different populations [28]. Also, in our study, baseline definition in terms of baseline value and time window show major consequences on AKI prevalence at ED. For each baseline time window, the most recent value might be the most accurate representation of the patient's premorbid renal function to determine AKI, and may reduce over-estimation of AKI diagnoses. Moreover, instead of evaluating all baseline definitions, only evaluating the most recent baseline time window may reduce the number of false positive diagnoses. In contrast, we show that including all guideline criteria as well as all baseline time windows increases the number of AKI cases, which may lead to over-estimation of AKI diagnoses at the ED.
Our study has several strengths. We used a large dataset with a well-documented population. This allowed us to study the AKI prevalence at the ED using real-world data reflecting current clinical care at the ED of several years. Furthermore, the use of a relational database, which continuously stores laboratory and clinical data for every patient, ensures maximum completeness and integrity of the data, which are often problems associated with retrospective data analyses.

This study also has several limitations. The current study is a retrospective single center study in an academic center, therefore these results may not be generalizable to other patient populations. Additional studies are necessary to determine the extent to which the definition of baseline affects AKI prevalence in other clinical settings. Furthermore, in the current literature, baseline $\mathrm{SCr}$ is defined as a measurement in a healthy person. By including ED visits of patients with at least one $\mathrm{SCr}$ value in their clinical history we may have introduced a selection towards sicker patients as they had their kidney function assessed previously. Defining criteria for baseline SCr that reflects kidney function in a stable healthy condition would be ideally 


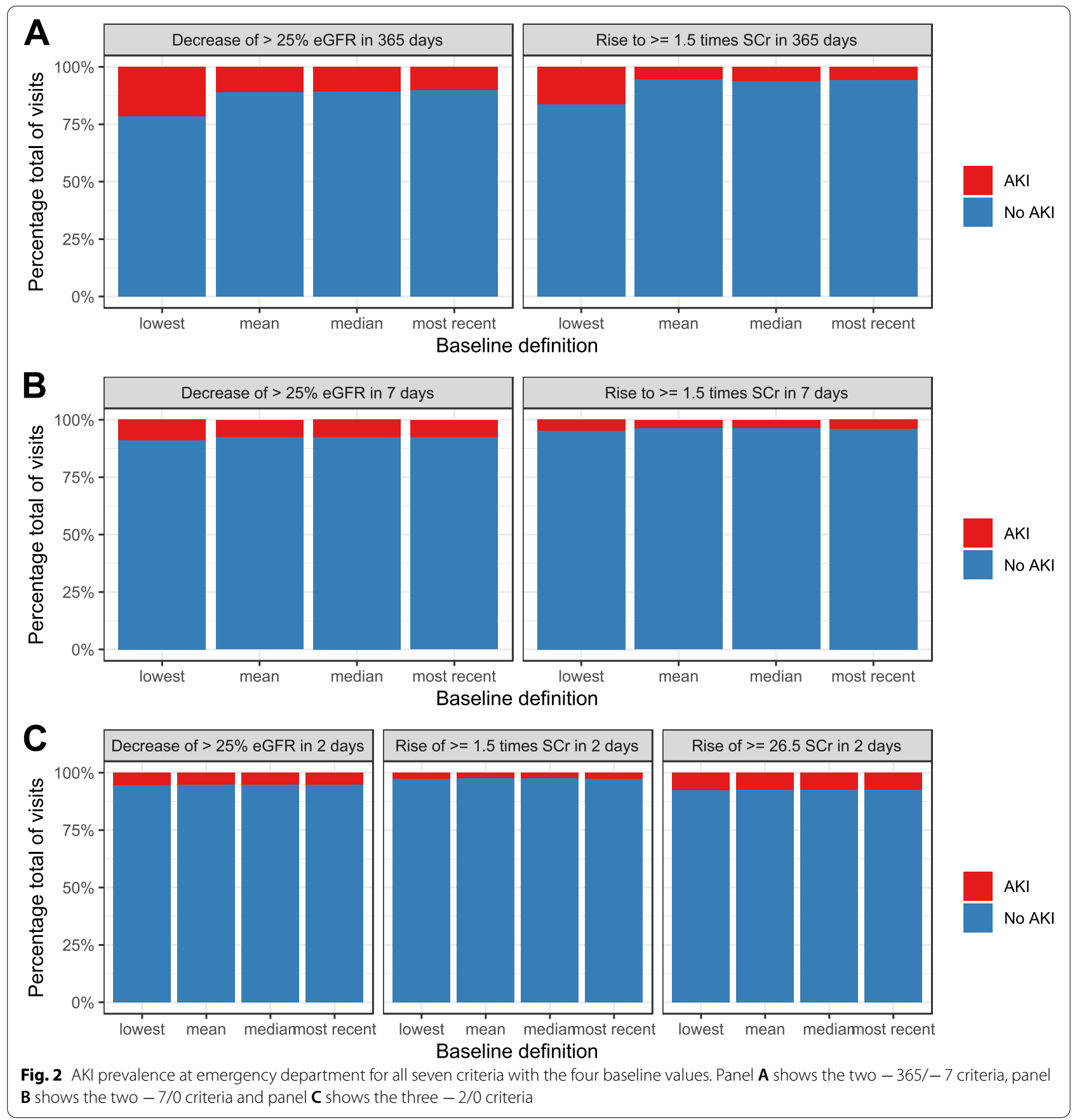

to compare the $\mathrm{SCr}$ value with the value at admission to the ED, and also for application of CDS [13]. However, as an academic hospital the majority of the patients with a blood test at $\mathrm{ED}$ receive a $\mathrm{SCr}$ test during routine visit, thereby reducing this bias in our analyses. In addition, the values we included were the available $\mathrm{SCr}$ values used by clinicians, providing a better reflection of clinical practice and the current AKI prevalence for diseased patients to explore the challenges of
AKI diagnosis at ED. Lastly, half of the ED visits either did not have a SCr-BL and/or a SCr-ED (results not shown). For this reason, we may have excluded patients with an elevated SCr during ED visit who may have had AKI. As a consequence, the computed rate of AKI diagnosis may not reflect the true AKI incidence at our ED. AKI incidence at ED estimated with the discussed guidelines may result in an underestimation of the true AKI incidence. 


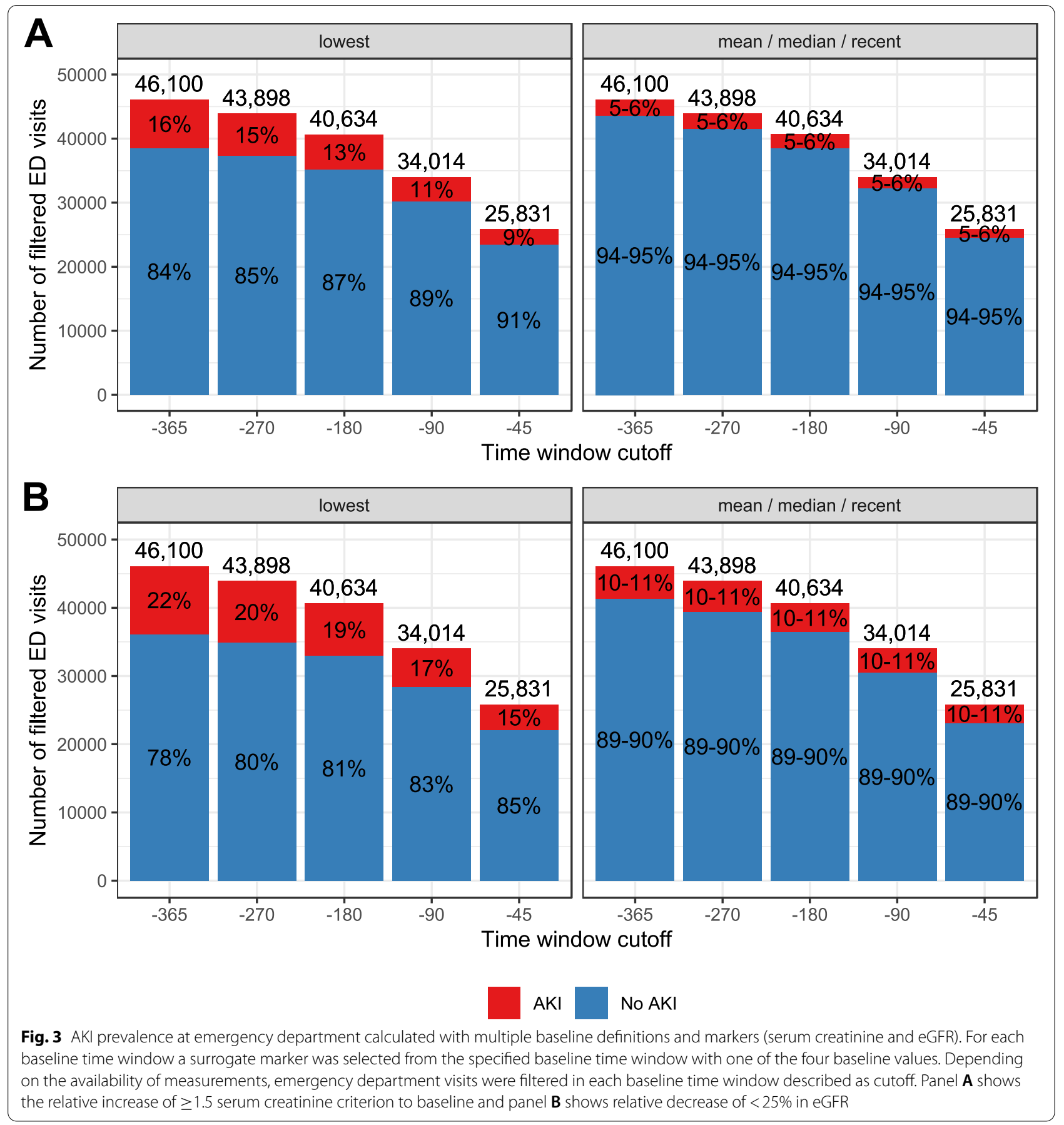

To compare the guidelines in terms of baseline definition, we adapted the criteria by selecting one measurement from the baseline time window. However, the AKIN criteria evaluate all $\mathrm{SCr}$ measurements from the $48 \mathrm{~h}$ prior to ED. Comparing all $\mathrm{SCr}$ measurements from this time window with the SCr-ED may have resulted in a higher AKI prevalence. However, regardless of baseline definition, AKIN AKI prevalence remained the same indicating that the majority of the visits only had one $\mathrm{SCr}$ measurement available, thereby reducing this bias in our analyses. As only a small proportion $(N=3322,7.0 \%)$ of all visits had at least one $\mathrm{SCr}$ measurement in the $48 \mathrm{~h}$ prior to ED visit, computing the prevalence on all patients result in a lower AKI prevalence at ED. Moreover, we did not exclude patients who revisited our center during the study inclusion time window. Recurrent 
Table 3 AKI prevalence at emergency department based on different guidelines

\begin{tabular}{|c|c|c|c|c|c|}
\hline Guideline & Baseline value & $\mathrm{N}$ visits & AKI (\%) & $\begin{array}{l}\text { AKI hospital admissions } \\
\text { (\%) }\end{array}$ & AKI KDIGO stage (\%) \\
\hline \multirow[t]{16}{*}{ Rifle } & Lowest & 47,373 & $11,354(24.0 \%)$ & $8524(75.1 \%)$ & $0: 3677(32.4 \%)$ \\
\hline & & & & & $1: 4903(43.2 \%)$ \\
\hline & & & & & 2: $1872(16.5)$ \\
\hline & & & & & 3: 902 (7.9) \\
\hline & Mean & 47,373 & $5476(11.6 \%)$ & $4478(81.8 \%)$ & 0: 2711 (49.5\%) \\
\hline & & & & & 1: 1889 (34.5\%) \\
\hline & & & & & 2: $567(10.4 \%)$ \\
\hline & & & & & 3: $309(5.6 \%)$ \\
\hline & Median & 47,373 & $5493(11.6 \%)$ & $4505(82.0 \%)$ & $0: 2424(44.1 \%)$ \\
\hline & & & & & 1: $2082(37.9 \%)$ \\
\hline & & & & & $2: 644(11.7 \%)$ \\
\hline & & & & & $3: 343(6.2 \%)$ \\
\hline & Most recent & 47,373 & $5234(11.0 \%)$ & 4340 (82.9\%) & $0: 2369(45.3 \%)$ \\
\hline & & & & & 1: 1935 (37.0\%) \\
\hline & & & & & 2: 601 (11.5\%) \\
\hline & & & & & 3: $329(6.3 \%)$ \\
\hline \multirow[t]{12}{*}{$\mathrm{AKIN}^{\mathrm{a}}$} & Lowest & 3322 & $256(7.7 \%)$ & $231(90.2 \%)$ & 1: 139 (54.3\%) \\
\hline & & & & & $2: 62(24.2 \%)$ \\
\hline & & & & & 3: 55 (21.5\%) \\
\hline & Mean & 3322 & $248(7.5 \%)$ & $223(89.9 \%)$ & 1: $192(77.4 \%)$ \\
\hline & & & & & $2: 30(12.1 \%)$ \\
\hline & & & & & $3: 26(10.5 \%)$ \\
\hline & Median & 3322 & $247(7.4 \%)$ & 222 (89.9\%) & 1: $189(76.5 \%)$ \\
\hline & & & & & $2: 30(12.1 \%)$ \\
\hline & & & & & 3: $28(11.3 \%)$ \\
\hline & Most recent & 3322 & $247(7.4 \%)$ & $223(90.3 \%)$ & 1: 197 (79.8\%) \\
\hline & & & & & 2: $23(9.3 \%)$ \\
\hline & & & & & 3: 27 (10.9\%) \\
\hline \multirow[t]{12}{*}{ KDIGO } & Lowest & 47,373 & $7694(16.2 \%)$ & $6023(78.3 \%)$ & 1: 4920 (63.9\%) \\
\hline & & & & & 2: $1872(24.3 \%)$ \\
\hline & & & & & 3: $902(11.7 \%)$ \\
\hline & Mean & 47,373 & 2798 (5.9\%) & $2430(86.8 \%)$ & 1: $1922(68.7 \%)$ \\
\hline & & & & & 2: 567 (20.3\%) \\
\hline & & & & & 3: 309 (11.0\%) \\
\hline & Median & 47,373 & 3105 (6.6\%) & 2664 (85.8\%) & $1: 2118(68.2 \%)$ \\
\hline & & & & & 2: 644 (20.7\%) \\
\hline & & & & & $3: 343(11.0 \%)$ \\
\hline & Most recent & 47,373 & 2914 (6.2\%) & $2550(87.5 \%)$ & 1: $1984(68.1 \%)$ \\
\hline & & & & & 2: 601 (20.6\%) \\
\hline & & & & & 3: 329 (11.3\%) \\
\hline
\end{tabular}

Prevalence shown as percentages between brackets was calculated on visits with at least one baseline value and a serum creatinine measurement at emergency department visit $(N=47,373)$. The most recent baseline definition was used in combination with the $-365 /-7$ days time window to diagnose AKI. Number and percentage of hospital admission and KDIGO stage are shown for patients with AKI according to the guideline

${ }^{a}$ As the AKIN criteria only evaluate the $48 \mathrm{~h}$ prior to ED visit, only visits with measurements within this period were used to calculate the AKI prevalence $(N=3322)$

patients may have more complications that might be associated with a higher chance of AKI. As a result, this may have influenced the AKI prevalence. However, evaluating the AKI prevalence at the ED requires the complete
ED population, including repetitive visits of not-critically ill patients.

Multiple additions can be made to the guidelines to improve diagnosis. For example, the CKD-EPI formula 
may provide inaccurate estimations of kidney function in patients as this formula was developed on healthy patients of which the concentration of $\mathrm{SCr}$ was in steadystate. As a consequence, GFR estimates may be over- or underestimated that may affect AKI diagnosis. Dynamic eGFR formulas such as suggested by Chen et al. (2013) may provide a better estimate of GFR [29]. Moreover, the addition of novel biomarkers for AKI diagnosis, such as neutrophil gelatinase-associated lipocalin (NGAL), cycle arrest biomarkers, tissue inhibitor metalloproteinase-2 (TIMP-2) and insulin-like growth factor-binding protein (IGFBP7), may be added in the future to the guidelines to improve AKI diagnosis [30].

Importantly, our data provide evidence that researchers and developers of CDS systems and machine learning algorithms based on different baseline definitions for AKI criteria could account for major changes on the diagnosis of AKI when their research is implemented in the ED setting. The use of different definitions for baseline may thus lead to delayed recognition and underestimation of AKI and affect the clinical course and initiation of therapy in the early stages of AKI. As more hospitals implement AKI guidelines for automated AKI diagnosis, being familiar with the consequences of ambiguous baseline definitions is of upmost importance. As large routine care datasets facilitate retrospective research and prospective implementation of clinical decision support systems, researchers as well as CDS developers should carefully weigh the pros and cons of different definitions, criteria and guidelines in terms of false positive or false negative diagnoses in multidisciplinary teams to tailor the definition of AKI to their needs [31].

\section{Conclusions}

In conclusion, this study shows that ambiguous definitions for baseline can have major consequences on the AKI diagnosis in patients presenting at the ED. Incorrect definition of baseline may result in misdiagnosis of AKI patients at the ED with suboptimal decisions for treatment and medication as result. Clinicians, as well as researchers and developers of automatic diagnostic tools such as clinical decision support systems should take these considerations into account when aiming to diagnose AKI in clinical and research settings.

\footnotetext{
Abbreviations

AKI: Acute kidney injury; ED: Emergency department; SCr: Serum creatinine; SCr-ED: Serum creatinine concentration at emergency department visit; SCrBL: Baseline serum creatinine concentration; EHR: Electronic health records; CDS: Clinical decision support; eGFR: Estimated Glomerular Filtration Rate; UMCU: University Medical Center Utrecht; UPOD: Utrecht Patient Orientated Database.
}

\section{Supplementary Information}

The online version contains supplementary material available at https://doi. org/10.1186/s12882-021-02581-x.

Additional file 1: Supplementary data Table 1. Frequency table of the number of emergency department visits of all 20,488 included patients. Supplementary data Table 2. Mean and standard deviation of baseline (BL) serum creatinine (SCr) and eGFR for each baseline time window and baseline value. Supplementary data Table 3. AKI prevalence for each of the seven criteria combined with each baseline value. Supplemental Figure 1. Boxplots of delta serum creatinine $(\mathrm{SCr})$ between the selected baseline $\mathrm{SCr}$ value and the $\mathrm{SCr}$ measurement at emergency department visit, for each baseline definition. Supplemental Figure 2. Boxplots of delta glomerular filtration rate (eGFR) between the selected baseline eGFR value and the eGFR measurement at emergency department visit, for each baseline definition.

\section{Acknowledgements}

NA

\section{Authors' contributions}

$\mathrm{MN}, \mathrm{MK}, \mathrm{WT}, \mathrm{JU}, \mathrm{KK}$ and SH contributed to the conception and the design of the study. MN and SH collected and assembled the data. All authors contributed to the analysis and interpretation of the results. All authors read and approved the final manuscript. Manuscript writing: all authors. Final approval of manuscript: all authors. Accountable for all aspects of the work: all authors.

\section{Funding}

No funding.

\section{Availability of data and materials}

The data underlying this article cannot be shared publicly due to ethical and privacy reasons for the privacy of individuals that participated in the study. The data will be shared on reasonable request to the corresponding author.

\section{Declarations}

\section{Ethics approval and consent to participate}

This study was performed in accordance with the declaration of Helsinki and the ethical guidelines of our institution. The institutional review board of the UMC Utrecht approved this study (reference number 19-042/C) and waived the need for informed consent as only pseudonymized data were used for this study. Data collection and handling was conducted in accordance with European privacy legislation (GDPR).

\section{Consent for publication}

Not required.

\section{Competing interests}

M.S.A. Niemantsverdriet is supported by a PhD fellowship from SkylineDx BV, Rotterdam and being employed by SkylineDx BV, Rotterdam. D.B. Bellomo is being employed by SkylineDx BV, Rotterdam. S. Haitjema is supported by a fellowship of Abbott Diagnostics. All other authors report no conflicts of interest.

\section{Author details}

${ }^{1}$ Central Diagnostic Laboratory, University Medical Center Utrecht, Utrecht University, Room Number G03.551, UMC Utrecht, Heidelberglaan 100, Utrecht 3584 CX, The Netherlands. ${ }^{2}$ SkylineDx, Lichtenauerlaan 40, Rotterdam 3062 ME, The Netherlands. ${ }^{3}$ Department of Nephrology and Hypertension, University Medical Center Utrecht, Utrecht University, Heidelberglaan 100, Utrecht 3584 CX, The Netherlands. ${ }^{4}$ Department of Internal Medicine, University Medical Center Utrecht, Utrecht University, Heidelberglaan 100, Utrecht 3584 CX, The Netherlands.

Received: 22 June 2021 Accepted: 25 October 2021 Published online: 08 November 2021 


\section{References}

1. Ronco C, Bellomo R, Kellum JA. Acute kidney injury. Lancet. 2019;394(10212):1949-64.

2. James MT, Hobson CE, Darmon M, Mohan S, Hudson D, Goldstein SL, et al. Applications for detection of acute kidney injury using electronic medical records and clinical information systems: workgroup statements from the 15th ADQI Consensus Conference. Can J Kidney Health Dis. 2016;3:100

3. Pickup L, Loutradis C, Law JP, Arnold JJ, Dasgupta I, Sarafidis P, et al. The effect of admission and pre-admission serum creatinine as baseline to assess incidence and outcomes of acute kidney injury in acute medical admissions. Nephrol Dial Transplant. 2021. https://doi.org/10.1093/ndt/ gfaa333.

4. Lewington AJP, Cerdá J, Mehta RL. Raising awareness of acute kidney injury: a global perspective of a silent killer. Kidney Int. 2013;84(3):457-67.

5. Mehta RL, McDonald B, Gabbai F, Pahl M, Farkas A, Pascual MTA, et al. Nephrology consultation in acute renal failure: does timing matter? Am J Med. 2002;113(6):456-61.

6. Bellomo R, Ronco C, Kellum JA, Mehta RL, Palevsky P. Acute renal failure - definition, outcome measures, animal models, fluid therapy and information technology needs: the Second International Consensus Conference of the Acute Dialysis Quality Initiative (ADQI) Group. Crit Care. 2004;8(4):1-9.

7. Mehta RL, Kellum JA, Shah SV, Molitoris BA, Ronco C, Warnock DG, et al. Acute kidney injury network: report of an initiative to improve outcomes in acute kidney injury. Crit Care. 2007;11(2):1-8.

8. Kellum JA, Lameire N, Aspelin P, Barsoum RS, Burdmann EA, Goldstein SL, et al. Kidney disease: improving global outcomes (KDIGO) acute kidney injury work group. KDIGO clinical practice guideline for acute kidney injury. Kidney Int Suppl. 2012;2(1):1-138.

9. Bellomo R, Kellum JA, Ronco C. Acute kidney injury. Lancet. 2012;380(9843):756-66. https://doi.org/10.1016/S0140-6736(11)61454-2.

10. Tomašev N, Glorot X, Rae JW, Zielinski M, Askham H, Saraiva A, et al. A clinically applicable approach to continuous prediction of future acute kidney injury. Nature. 2019;572(7767):116-9. https://doi.org/10.1038/ s41586-019-1390-1.

11. Medic G, Kließ MK, Atallah L, Weichert J, Panda S, Postma M, et al. Evidence-based clinical decision support systems for the prediction and detection of three disease states in critical care: a systematic literature review. F1000Research. 2019;8:1728.

12. Thongprayoon C, Hansrivijit P, Kovvuru K, Kanduri SR, Torres-Ortiz A, Acharya $P$, et al. Diagnostics, risk factors, treatment and outcomes of acute kidney injury in a new paradigm. J Clin Med. 2020;9(4):1104.

13. Gaião S, Cruz DN. Baseline creatinine to define acute kidney injury: is there any consensus? Nephrol Dial Transplant. 2010;25(12):3812-4.

14. Pan HC, Chien YS, Jeng CC, Tsai MH, Fan PC, Chang CH, et al. Acute kidney injury classification for critically ill cirrhotic patients: a comparison of the KDIGO, AKIN, and RIFLE classifications. Sci Rep. 2016;6(March):1-9.

15. Holmes J, Geen J, Phillips B, Williams JD, Phillips AO. Community acquired acute kidney injury: findings from a large population cohort. QJM. 2017;110(11):741-6.

16. Selby NM, Crowley L, Fluck RJ, Mclntyre CW, Monaghan J, Lawson N, et al. Use of electronic results reporting to diagnose and monitor AKI in hospitalized patients. Clin J Am Soc Nephrol. 2012;7(4):533-40.
17. Kellum JA, Prowle JR. Paradigms of acute kidney injury in the intensive care setting. Nat Rev Nephrol. 2018;14(4):217.

18. Ten Berg MJ, Huisman A, Van Den Bemt PMLA, Schobben AFAM, Egberts ACG, Van Solinge WW. Linking laboratory and medication data: new opportunities for pharmacoepidemiological research. Clin Chem Lab Med. 2007;45(1):13-9.

19. Levey AS, Stevens LA, Schmid CH, Zhang Y, Castro AF, Feldman HI, et al. A new equation to estimate glomerular filtration rate. Ann Intern Med. 2009:150(9):604-12

20. Siew ED, Matheny ME, Ikizler TA, Lewis JB, Miller RA, Waitman LR, et al. Commonly used surrogates for baseline renal function affect the classification and prognosis of acute kidney injury. Kidney Int. 2010;77(6):53642. https://doi.org/10.1038/ki.2009.479.

21. Hoste EAJ, Kellum JA, Selby NM, Zarbock A, Palevsky PM, Bagshaw SM, et al. Global epidemiology and outcomes of acute kidney injury. Nat Rev Nephrol. 2018;14(10):607-25. https://doi.org/10.1038/s41581-018-0052-0.

22. Wonnacott A, Meran S, Amphlett B, Talabani B, Phillips A. Epidemiology and outcomes in community-acquired versus hospital-acquired aki. Clin J Am Soc Nephrol. 2014;9(6):1007-14.

23. Scheuermeyer FX, Grafstein E, Rowe B, Cheyne J, Grunau B, Bradford A, et al. The clinical epidemiology and 30-day outcomes of emergency department patients with acute kidney injury. Can J Kidney Health Dis. 2017:4:2054358117703985.

24. Levi TM, de Souza SP, de Magalhães JG, de Carvalho MS, Cunha ALB, de Oliveira Dantas JGA, et al. Comparison of the RIFLE, AKIN and KDIGO criteria to predict mortality in critically ill patients. Rev Bras Ter Intensiva. 2013;25(4):290

25. Ülger F, Küçük MP, Küçük AO, Illkaya NK, Murat N, Bilgiç B, et al. Evaluation of acute kidney injury (AKI) with RIFLE, AKIN, CK, and KDIGO in critically ill trauma patients. Eur J Trauma Emerg Surg. 2018;44(4):597-605.

26. Wald R. Predicting baseline creatinine in hospitalized patients. Clin J Am Soc Nephrol. 2012;7(155-9041):5.

27. Makris K. The role of the clinical laboratory in the detection and monitoring of acute kidney injury. J Lab Precis Med. 2018;3:69.

28. De Rosa S, Samoni S, Ronco C. Creatinine-based definitions: from baseline creatinine to serum creatinine adjustment in intensive care. Crit Care. 2016;20(1):1-5

29. Chen S. Retooling the creatinine clearance equation to estimate kinetic GFR when the plasma creatinine is changing acutely. J Am Soc Nephrol. 2013;24(6):877-88.

30. Albert C, Haase M, Albert A, Zapf A, Braun-Dullaeus RC, Haase-Fielitz A. Biomarker-guided risk assessment for acute kidney injury: time for clinical implementation? Ann Lab Med. 2020;41(1):1-15.

31. Bezemer T, De Groot MCH, Blasse E, Ten Berg MJ, Kappen TH, Bredenoord $\mathrm{AL}$, et al. A human (e) factor in clinical decision support systems. J Med Internet Res. 2019;21(3):e11732.

\section{Publisher's Note}

Springer Nature remains neutral with regard to jurisdictional claims in published maps and institutional affiliations.

Ready to submit your research? Choose BMC and benefit from

- fast, convenient online submission

- thorough peer review by experienced researchers in your field

- rapid publication on acceptance

- support for research data, including large and complex data types

- gold Open Access which fosters wider collaboration and increased citations

- maximum visibility for your research: over 100M website views per year

At $\mathrm{BMC}$, research is always in progress.

Learn more biomedcentral.com/submissions 